\title{
Precession Electron Diffraction Detection and Phase Mapping of Retained Austenite and Carbides in a Heat Treated Low Alloy Carbon Steel Using a JEOL ARM 200 TEM with an AppFive Topspin System for Synchronized Beam Scanning and Precession
}

\author{
John Mardinly ${ }^{1}$, Amith Darbal ${ }^{2}$ \\ 1. LeRoy Eyring Center for Solid State Science, Arizona State University, Tempe, AZ USA. \\ 2. AppFive LLC, Tempe, AZ USA.
}

Retained austenite in heat treated steels is usually measured using X-Ray diffraction, with protocols outlined in ASTM Standard E975 [1]. Retained austenite has been measured in high alloy steels with large grains of retained austenite using EBSD [2]. However, low alloy steels heat treated to reduce retained austenite may have small grains of retained austenite that cannot reliably be detected by EBSD [3] or X-Ray diffraction.

A steel alloy of composition: $0.5 \%$ Carbon, $1.12 \%$ Chromium, $1.6 \%$ Tungsten, $1 \%$ Silicon, $0.35 \%$ Manganese, $0.15 \%$ Vanadium and balance Iron was submitted to our laboratory for analysis. The specimen was fully austenitized at $900 \mathrm{C}$, quenched to room temperature then to $-200 \mathrm{C}$ at which temperature it was held for 48 hours. Finally, it was annealed at $225 \mathrm{C}$ for three hours.

Retained austenite was measured using X-ray Diffraction using protocols outlined in ASTM Standard E975 [1]. There was no retained austenite detected.

A slice of the steel was prepared for TEM by standard dimple and ion milling techniques.

Data acquisition was in a JEOL ARM 200 Probe-Corrected TEM with a Schottky Field Emission source operated at $200 \mathrm{KeV}$ in TEM mode, with an AppFive Topspin system for synchronized beam scanning and precession. The microscope was operated in TEM alpha-L mode, spot size 5 using a 10 micron condenser aperture. Since the microscope was operated in TEM mode, the CEOS probe corrector did not influence the probe size. This choice of optical modes was driven by the desire to use a highly parallel beam for electron diffraction, rather than the highly convergent beam that results from using the probe corrector in STEM mode. The electron diffraction patterns were collected using a Sting Ray Firewire camera focused onto the fluorescent screen of the microscope. This camera collected patterns $144 \times 144$ pixels, in 0.01 seconds per pattern. The field of view was 292x301 nanometers. 92,700 electron diffraction patterns were collected from the field of view in 15 minutes. Each pixel was approximately one nanometer in size.

Post acquisition analysis was completed using Astar data analysis software which uses the template method of Rauch et al [3]. Electron diffraction patterns were calculated for every zone axis for ferrite, austenite and all five carbides listed in ASTM Standard E975 [1]. Matching the templates from 7 phases to the 92,700 patterns acquired is very computationally intensive, and took over 5 hours on a $3.1 \mathrm{GHz}$ Core $7 \mathrm{CPU}$ with 64 bit Windows 7, 16GB of RAM and a 250GB solid state drive.

[1] American Society for Testing Materials, 15 Inverness Way East, Englewood CO 80112. 
[2] M.Yaso, S. Hayashi and S. Morito, Materials Transactions, Japan Inst. of Metals, Vol. 50 No.2 (2009) pp. 275-279.

[3] E. Rauch, M. Veron, J. Portillo, Microscopy and Analysis Nanotechnology Supplement, Nov. 2008.

The authors gratefully acknowledge the use of the facilities within the LeRoy Eyring Center for Solid State Science at Arizona State University.

The authors gratefully acknowledge Seyed Nematollah Ahmadyan at the University of Illinois for supplying the steel specimen.
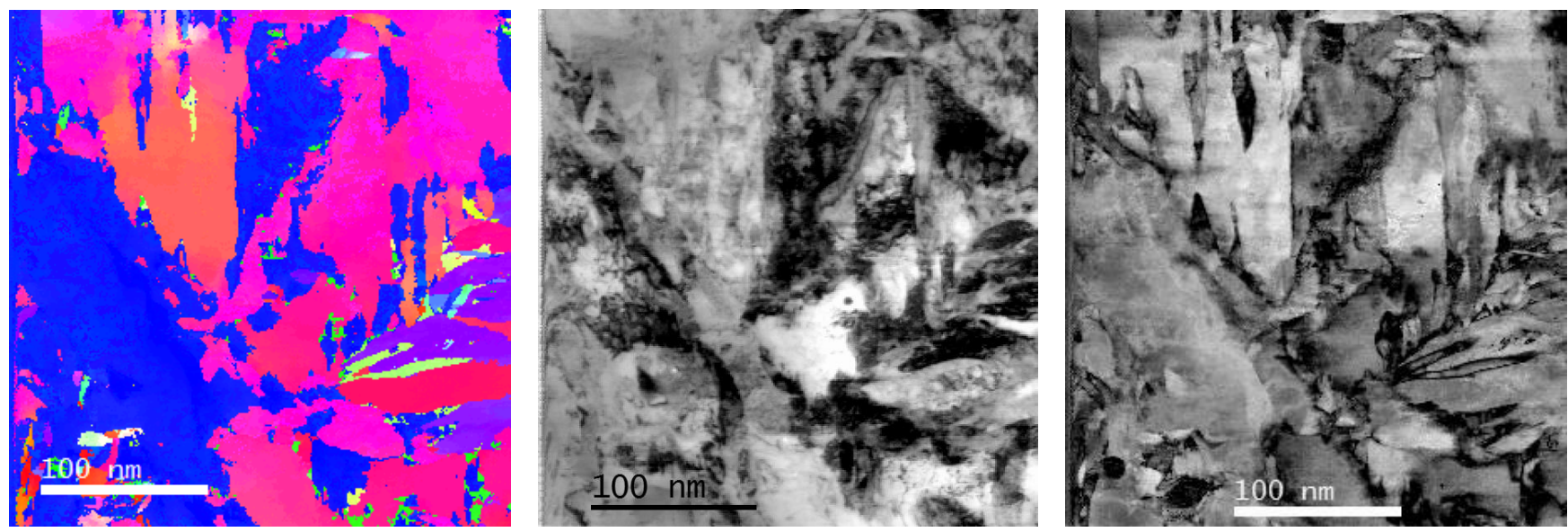

Figure 1. Orientation Map, Virtual Brightfield Image and Reliability Map.

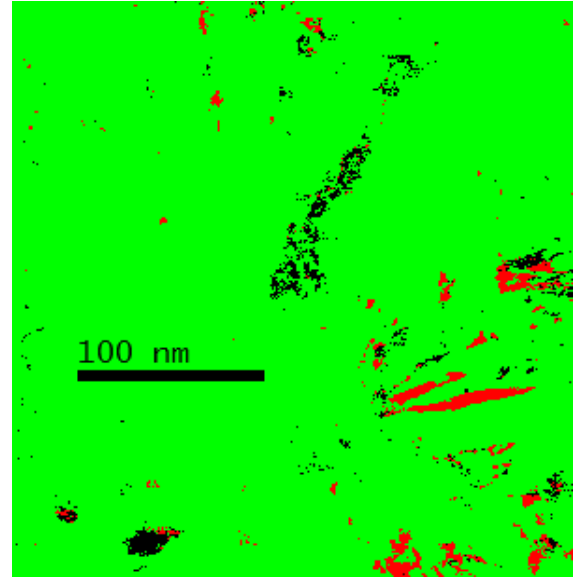

A

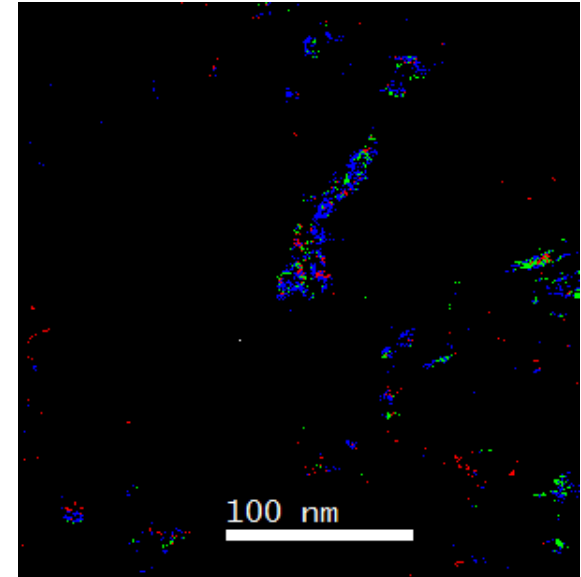

B

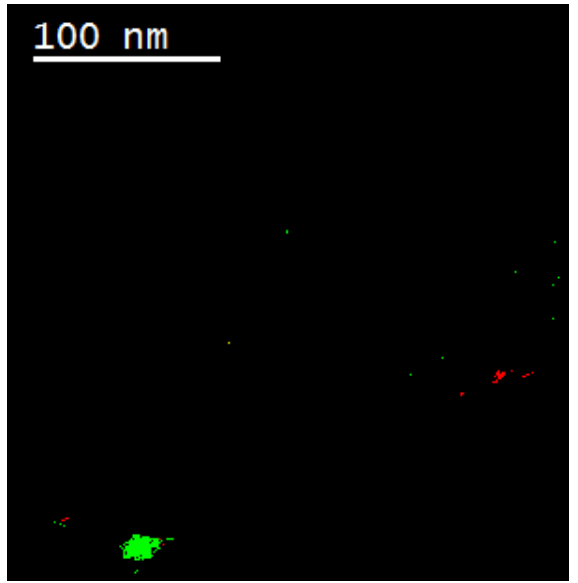

C

Figure 2. Phase Maps for A: Ferrite (green) and Austenite (Red), B: Cr23C6 (green), Cr7C3 (blue) $\mathrm{Fe} 3 \mathrm{C}$ (red) and $\mathbf{C}$ : $\mathrm{Cr} 3 \mathrm{C} 2$ (red) plus Fe6C (green). 